\title{
La impronta de Vicente Riva Palacio en las exposiciones de arte mexicano
}

Carlos A. Molina P.

Estas páginas discurren sobre el origen y persistencia de dos maneras de pensar el concepto de Museo Nacional en México. Busco apuntar a las influencias que puedan hallarse en diversas puestas en escena cuyo asunto era la cultura mexicana entre 1877 y 1952 . Propongo que dichas configuraciones responden a dos influjos discursivos; uno de índole histórica y narrativa, el otro formalista y estetizante. Contrasto entonces el pormenor de etapas para el desarrollo de la patria con otra preocupación que está en los valores plásticos como directrices para el trabajo expositivo. La intención es señalar a tres instantes en que la práctica en el museo se revisa y se transforma.

En la particular disposición de objetos que caracterizaba al Salón de Monolitos se observa un ejercicio reduccionista de la historia.

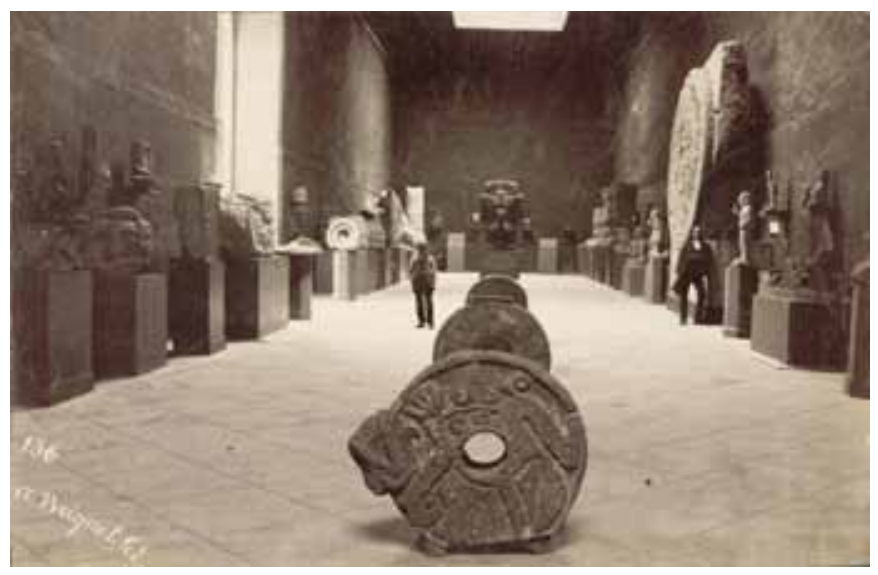

Allí existe un esquema museográfico a partir de conjuntos mayores desde la similitud entre objetos y que opera estabilizando sus significados. Esa sala muestra al conjunto de esculturas que contiene como una versión totalizadora de la heredad prehispánica para el presente, y a su vez se pretende explicación de aquél pasado. Hay otro modo de concentrar y exhibir objetos en un museo y cuya racionalidad es narrativa, es decir, cuenta una historia. 


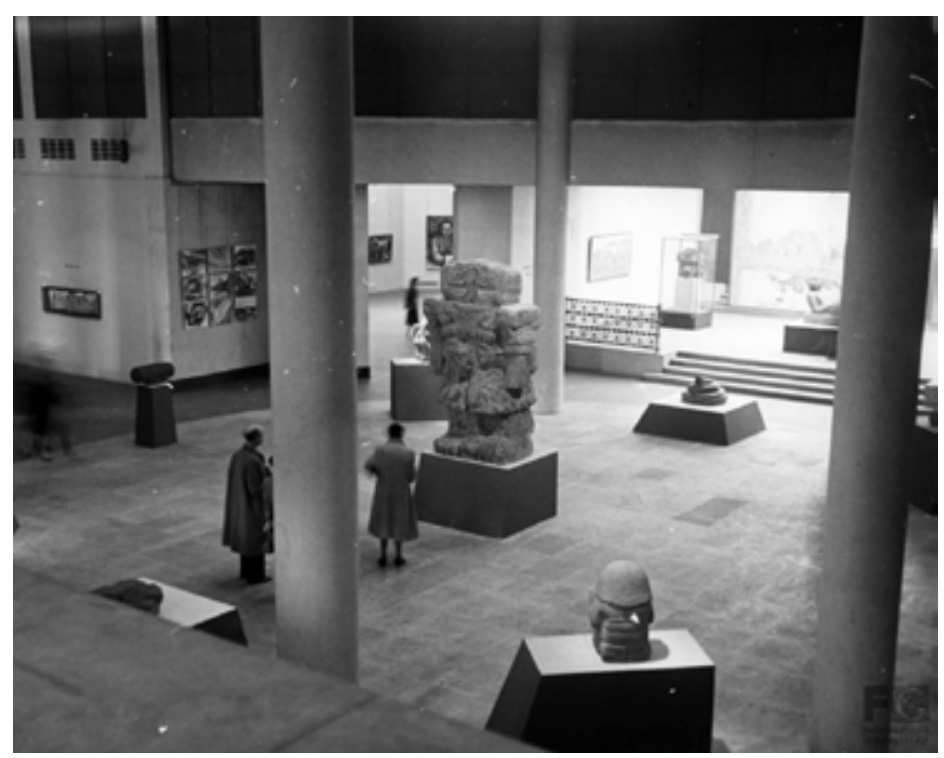

Ese es el que se ve en la Exposición de Arte Moderno y Antiguo de México entre 1950 y 1953 . Aquí, primero ocurre una separación de los objetos de su contexto histórico específico para proponerlos como parte de un absoluto de Arte atemporal y universal. Luego y haciendo caso omiso de sus particularidades históricas, se integra a la muestra completa en una narrativa tripartita específica. Así, todas las partes refieren a un meta-texto y en tanto que pertenecientes a él, al México así explicado, tienen sentido (me refiero a este esquema con la ecuación Nacimiento + Resistencia + Consolidación $=$ México) Como lo ha explicado Eugenio Donato la característica esencial del tipo de conocimiento que un museo produce, existe sólo en tanto que puede borrar la aparente heterogeneidad de los objetos que aloja y volverse una unidad coherente desde los enunciados que sus museógrafos o curadores articulan a partir del conjunto (1). La tipología simple que aquí adelanto se pregunta de qué manera los objetos expuestos sirven como punto de encuentro entre la abstracción de mexicanidad y el trabajo concreto de los mexicanos que la enuncian. Para ello, creo, sería pertinente volver a mirar la figura de Vicente Riva Palacio.

Vicente Riva Palacio es esencial para entender cómo, a lo largo del siglo diecinueve la primer y urgente tarea del estado en México es volverse una entidad operativa y consolidar su aparato legal-administrativo. 


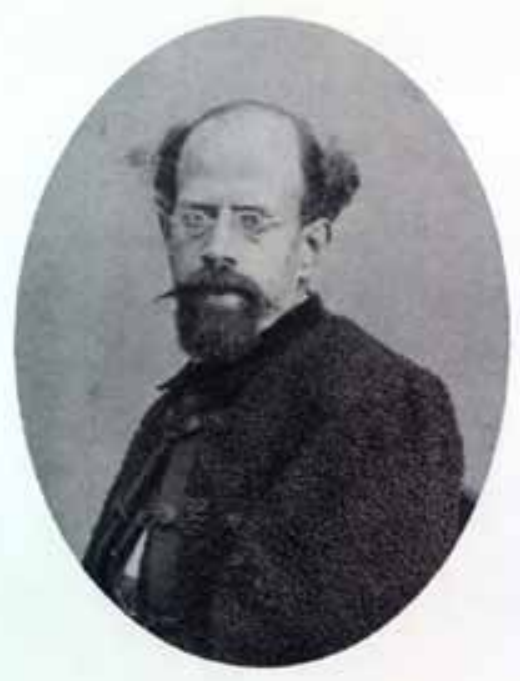

Su padre, el primer regidor del Ayuntamiento de México en 1830 y Ministro de Justicia en 1851, pondrá firma en el primer decreto que mandata la creación de un Museo Nacional ${ }^{2}$. La revista El Museo mexicano, ó, Miscelanea pintoresca de amenidades curiosas é instructivas era publicada entre 1842 y 1845 por Ignacio Cumplido, un amigo de don Mariano. Redactor de periódicos y dramaturgo, Vicente Riva Palacio tiene muy claro cuál es el poder de la opinión pública (se le supone incluso el fundador de esa rancia gracia mexicana que es la sátira política). En toda instancia dice explícitamente estar hablando: "única y exclusivamente al pueblo"3. . Gobernador con Juárez e incontestable organizador del ejército que resistirá desde Zitácuaro, será perseguido por Lerdo en 1874. Con ese prestigio va a la causa del primer Díaz en Tuxtepec. Ministro de Fomento promoverá el rescate de Palenque y coloca sobre Reforma las estatuas de Colón (Carlos Cordier) y Cuauhtémoc (Miguel Noreña). Llegado el momento de la elección rumbo a la presidencia en 1880, elude esa gran decisión política y propone una Exposición Universal Mexicana. En fin, que el "museo" es preocupación constante toda su vida.

Pero cuando RivaPalacio dice "museo" se refiere no solamente al establecimiento educativo que exhibe y explica objetos de interés cultural, piensa también en lo que Thomas Richards ha definido como el "complejo archivístico". Es decir, un espacio utópico para el conocimiento comprensivo y que ubica un dominio geopolítico, el centro de todo conocimiento posible sobre la nación, la librería última y biblioteca de bibliotecas, el museo final. ${ }^{4}$ Existe una regla de correspondencia entre todo practicante de la curaduría y la articulación del complejo archivístico 5 . Al final de su vida, cuando RivaPalacio publica y supervisa la edición de México a través de los Siglos, la obra misma es la materialización 
de esa preocupación por lograr la supremacía del estado secular y por la que había luchado toda su vida. Si hay un proyecto que emerja por encima de sucesivos regímenes y sus signos a lo largo del siglo diecinueve, ese es justamente la premisa ideológica liberal cuya visión de mejoras sociales y económicas está predicada por una fe fiducia en la educación como herramienta. Esta enciclopedia de la mexicanidad, esta primer versión oficial de la historia del país, es advocación textual, instanciación literaria del mismo objetivo: el muestrario y explicación últimas de la nación. De allí se elucida la misma conclusión que de visitar la Galería de Monolitos tras 1877, lo indígena precolombino, su mezcla con el sino cristiano y la impronta del Progreso, explican lo nacional mexicano. ${ }^{6}$ El museo entonces es la materialización de una voluntad de los intelectuales mexicanos por ubicar una meta o circunstancia ideal, un objetivo que la nación está siempre por alcanzar.7

En 1882 RivaPalacio se lanza a fundar el Ateneo Mexicano de Artes y Ciencias. Es allí que encuentra coyuntura fundamental, a la vista está el culmen de la realización para la patria mexicana y le urge un modo de dar por afianzados esos logros. Un año más tarde sin embargo, está en la prisión militar de Tlatelolco y desde ahí escribe el segundo tomo de México a través de los siglos. Irineo Paz será el primero en hacernos notar que aquél trabajo como editor de Riva Palacio iba mucho más allá de la recolección y primer lectura de textos: "la Historia variaba en cuanto a la forma y al estilo... pero, en cuanto al fondo, pues las ideas que en ella se desarrollan son las mismas del Sr. Riva Palacio".

Si hacemos caso de Baxandall y en la explicación histórica de las imágenes existe una crítica inferencial, que habla más de los enunciados que acompañaron a esas series iconográficas llamadas museo que de la obra misma, entonces tiene todo el sentido del mundo que miremos con atención quién compuso aquél discurso y sobre qué presupuestos. La exposición se convierte así en una suerte de mise-en-scéne (puesta en escena) del patrimonio artístico-nacional cuyo rasgo saliente es reconocer al indígena como heredero biológico de una tradición que la nación moderna apropia para sí misma. Dicha declaración concierne a la Mexicanidad; es decir que el estudio de aquella práctica curatorial o museográfica implica tanto la localización de obras artísticas como sus usos, estructurando discursos sobre la nacionalidad. Su gramática deriva tanto de un tropo específico para explicar la historia, como de reglas visuales y artilugios de diseño que sirven para el acomodo de dichos objetos en el ámbito del museo. Tal proposición está necesariamente imbricada de los significantes Museo, Nación, Colección Científica, Vestigios Arqueológicos y Patrimonio Artístico.

El Museo Nacional para RivaPalacio es una versión de México compuesta por y para sus propios ciudadanos, que habría de ser consumida tanto por connacionales como extranjeros. Y esa "ficción del ser" puede solamente tener una 
narrativa de capitulado tripartita ${ }^{8}$. Resulta entonces que la construcción de la identidad se hace desde la recuperación, estudio y exhibición de elementos (en aquella época pensados como "especímenes científicos") y que se proponían como la herencia del México remoto. Tal muestra está articulada por objetos que, además, ilustran la "verdadera" esencia de lo mexicano y cuál ha de ser su representación. La identidad que los mexicanos modernos han construido para sí mismos incluye un pasado aborigen precolombino, así como una evaluación del presente, ahí donde se le enuncie. Es esa retórica a la que me refiero como particular del estado-nación mexicano, una narrativa de etapas simbólicas, sucesivas y necesarias, la ecuación implícita en RivaPalacio (Nacimiento, Resistencia y Consolidación). ${ }^{9}$
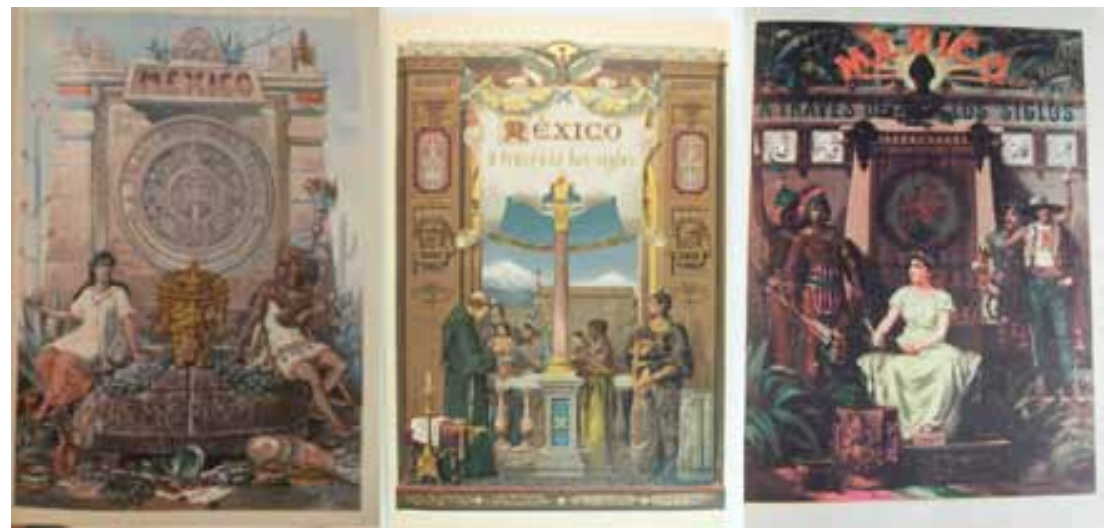

Este patrón para narrar la historia del país desde el museo resulta ser una estrategia empleada consistentemente en los dos siglos en que discursos sobre la modernidad, la nacionalidad y el patrimonio, se han elaborado en México. ${ }^{10}$

El capitulado de México a través de los Siglos

NACIMIENTO lo indígena y propio

Tomo I: "Historia antigua y de la conquista" (desde la antigüedad hasta 1521) Alfredo Chavero

RESISTENCIA contra España, USA '36 y '48 y Francia '61

Tomo II: "Historia del virreinato" (1521 - 1807) Vicente Riva Palacio

Tomo III: "La guerra de independencia" (1808 - 1821) Julio Zárate

Tomo IV: "México independiente" (1821 - 1855) Juan de Dios Arias + E. de Olavarría y Ferrari

Tomo V: “La reforma” (1855 - 1867) José María Vigil

CONSOLIDACIÓN el aquí y ahora...

Para la creación del Museo Nacional en 1822 Agustín de Iturbide dio el primer paso al decretar fundado el Conservatorio de Antigüedades y Gabinete de 
Historia Natural. Posteriormente Lucas Alamán planteó un Museo "que presentara una visión lo más completa posible del país, para lo cual debía incluir tres ramos: Antigüedades, Productos de la Industria, e Historia Natural"11. Esta propuesta atendía a un patrimonio cultural que ya había interesado a eruditos dentro y fuera del país (el presente o momento posterior a la Resistencia). Asimismo pretendía fomentar la producción artesanal (el reconocimiento del pasado indígena como consustancial al Nacimiento), enalteciendo las manufacturas de mejor calidad y mostrando algunas de las extranjeras susceptibles de ser producidas aquí mismo. Por último, quería mostrar la flora y fauna, especialmente aquella que pudiera redituar económicamente de alguna manera (el futuro o Consolidación inminente). Los afanes pedagógicos que se desprenden de la manera en que el líder Conservador concibió al Museo forman parte del amplio proyecto de construcción de la nacionalidad. Aunque de signo político opuesto, en ésta primera elaboración se lee el mismo credo histórico que en RivaPalacio.

Admitir una abstracción, tal como el "Arte" o la "Mexicanidad", es teleológico, puesto que se explica al pasado y al presente en términos del futuro. Es decir, la narrativa de la nación requiere por necesidad conciencia, volición o propósito a priori para su propia implementación. Para creer en la "consolidación" se requiere de momentos retrospectivos de "resistencia" y un mito del origen o "nacimiento". Si la narrativa fuera lineal, entonces no sería cohesiva ni formaría una totalidad, dado que presupone siempre un componente faltante (el nexo ausente entre principio y final, el desenlace inadvertido que sigue al acto de ser contada). En el caso de RivaPalacio y el museo mexicano, éste es un componente que antecede al origen y que por lo tanto proyecta la narrativa hacia un futuro que no puede contener en sí misma. No obstante, resulta esa ser la garantía de cierta inminencia, un futuro tal que está siempre a punto de volverse realidad. El cuento que el museo repite, la narrativa histórica de México ahí puesta en marcha y cuya materia es el Arte o la Ciencia o la Arqueología, es una instancia intermedia entre los narradores o profesionales del museo y la gente (esa ciudadanía que el museo mexicano decreta como el deber-ser para un pueblo realmente existente) ${ }^{12}$.

RivaPalacio fue nombrado Ministro de Fomento, Colonización, Industria y Comercio en 1876 con Porfirio Díaz, desde allí promovió políticas para la protección y difusión de monumentos y zonas arqueológicas. Es en esa capacidad que estudia al circuito de exposiciones internacionales y negocia el fallido proyecto para la Mexicana de $1880 .{ }^{13}$ Luego de visitar la Exposición Centenaria de 1876 Gabriel Mancera -comisionado especial de la junta de exposiciones en los Estados Unidos, dirigirá en primera instancia sus informes a la oficina de V. RivaPalacio (era su Oficial Mayor). Los más importantes intelectuales asociados con el Museo Nacional eran científicos: Gumesindo Mendoza, era un farmacis- 
ta, Genaro García, ingeniero en minas, Jesús Sánchez, botánico; Manuel Urbina, médico cirujano, al igual que Antonio Peñafiel. De manera que los métodos empleados por el museo podían ser solamente los que la ciencia acostumbraba. El primer rasgo aparente de la clasificación Linneana es su formalismo evidente. Parecido al modo en que se coleccionaban mariposas o pájaros en un gabinete, hallamos cabezas de serpiente "Aztecas" ordenadas por tamaño y parecido en el patio del Museo Nacional (14). Inaugurado el 6 de julio de 1866, el museo se orientó principalmente a la arqueología y a la historia estableciéndose allí la Sociedad Mexicana de Historia Natural. Treinta años después, Jesús Sánchez y Alfonso L. Herrera habían publicado los catálogos para las colecciones de mamíferos (1894), aves (1895), reptiles y batracios (1895), peces (1896) e imitación en cristal de invertebrados (1897). Ejemplares provenientes de ahí fueron llevados a Nueva Orléans en 1884, a París en 1889 y a Chicago en 1893. Los documentos de aprobación a nivel ministerial para estos traslados y la creación del Observatorio Meteorológico y Astronómico Nacional, llevan todos estampada la misma firma: Vicente Riva Palacio.

Adicionalmente, el pensamiento racial de Riva Palacio es de hecho fundamento para su actuar político y su convicción nacionalista. Derivar de una clasificación del género humano, una categoría específicamente mexicana, supone para Riva Palacio dilucidar que un museo que presente a tal estirpe biológica implica también que si es representable entonces es que el tejido nacional está completo y la realidad a la que refiere no está imaginada sino que existe objetivamente ${ }^{15}$. Éste híbrido epistemológico que Riva Palacio instrumenta hace confluir la taxonomía cultural con la entidad geopolítica que habría de reproducirse en el museo o la exposición internacional. Mestizaje y nacionalidad son una y la misma cosa; el concreto "indio" y el abstracto "mexicano" se unifican. Riva Palacio citará a su gusto a Darwin en The Descent of Man para probar que la "raza indígena" está más evolucionada y concebir desde dichas licencias al proceso de consolidación del estado-nación que ocupa a su generación en el bando liberal. El objetivo final es la ciudadanización ilustrada donde las distinciones raciales han dejado de tener sentido y, como sea, el mexicano es protéico y ahora está en su umbral óptimo. Para Riva Palacio la nación mexicana es más que el alma como propugnaba Ernest Rénan, es de hecho una entidad biológica, un cuerpo. La evidencia que cree hallar es paleontológica y antropométrica, más sólida en términos científicos, que la fe patriótica a la que aduce el francés.

Manuel Orozco y Berra había sido Director Interino del Museo Nacional en 1864 y 1867. En colaboración con Gumesindo Mendoza, publicó los Anales del Museo Nacional y montó la Galería de Monolitos. En 1882 esta galería exhibía en el patio del número 47 de la Calle de Museo, 147 piezas arqueológicas y otros vestigios menores en el piso superior ${ }^{16}$. Como se denuncia amargamente en el 
primer catálogo del Museo: "Las colecciones del Museo Nacional, hasta el año de 1865, estuvieron colocadas en un local muy reducido, y mal iluminado, compuesto de dos salas del edificio conocido entonces con el nombre de Universidad y destinado hoy para Conservatorio de Música y Declamación... el Departamento de Antigüedades Mexicanas no tenía lugar para sus colecciones..." ${ }^{17}$. En 1881 la tipología acordada para ubicar objetos en el Museo Nacional suponía las siguientes categorías: "astronomía, cronología, mitología, culto, urnas, piedras del juego de pelota, monumentos conmemorativos, epigrafía, arquitectura, escultura y objetos diversos".18 Sólo hasta 1897, con la Ley del 11 de Mayo y que declaraba los monumentos arqueológicos "Propiedad Nacional", estas esculturas encontraron cobijo en la entonces joven profesión de la antropología ${ }^{19}$.

En "El surgimiento de los museos de historia natural" de 1915, Oliver Cummings Farrington, primer curador del Field Museum of Natural History en Chicago y editor invitado de la revista Science para ese otoño, dice que "preservar objetos de la naturaleza en un museo" es inequívoco signo de que la sociedad encargada de atender tal establecimiento "ha superado a la naturaleza y ahora comprende aquellos objetos para generaciones posteriores." En dicho discurso con el que acepta la presidencia de la American Association of Museums, explica cómo es que aquellos especímenes naturales se transforman en el museo en objetos artísticos dado el efecto que supone seleccionarlos por características estéticas para su exhibición y el hecho de que sistematizarlos siguiendo a Linneo, Cuvier y Buffon los comprenda en un correlato más complejo. Haciendo un sumario listado de los museos de historia natural existentes en Norteamérica, Farrington menciona al mexicano y dice: "excellent section in which important material is preserved". Dicha comunicación es importante porque contiene la primer mención de un cambio de paradigma que nos interesa. Farrington señala lo que se anuncia a continuación para la práctica museal: "The lesson taught by a single object no longer suffices; it must be represented, if posible, in its natural setting".

La Secretaria de Instrucción Pública contrató en 1907 a George E. Seler, entonces Jefe del Departamento de Colecciones Americanas en el Museo de Berlín. Su programa de trabajo entonces estaba enfocado al "estudio de antigüedades, es decir, su colección y exposición" (20). Se esperaba que produjera y adaptara un catálogo a las particularidades de la colección y el personal del Museo Nacional. Seler hizo un registro detallado, que resultó en fichas escritas donde se consignaban las medidas, ocurría una breve descripción, se hacía un dibujo aproximado, se listaba bibliografía relevante para cada objeto, se usaba el nombre indígena y el castellanizado, se explicaba el uso original, la "raza" indígena que lo producía, el año de su colecta y la entidad que lo había traído al Museo Nacional (21). Taxonomía Linneana sin más. 
Entre mayo y julio de 1887, Franz Boas y J.W. Powell sostuvieron una polémica en la revista Science sobre métodos para la clasificación de especímenes en museos de etnología. De las varias cartas y replicas que a la revista dirigieron se desprende un acuerdo fundamental y que resonaría en la práctica cotidiana arreglando y mostrando al público colecciones en Europa y en América. Powell y Boas admiten que la premisa básica es la "interpretación de similitudes" como método para exhibir objetos arqueológicos, especímenes de historia natural e ilustraciones de tipos antropológicos y etnias. Concurren además en que el museo es primero repositorio de cosas culturalmente excepcionales, acto seguido la materialización de un sistema o dispositivo epistemológico que en última instancia ha de emplearse como herramienta educativa y docente. Powell advierte a lo largo de la discusión que estos presupuestos convienen particularmente a materiales precolombinos y a la descendencia actual de aquellos pueblos. Ese es precisamente el trabajo que Franz Boas haría un cuarto de siglo después en México. Estas tribus de principios del siglo veinte que son ligadas con vestigios materiales de sus antepasados remotos, no tienen que convertirse en un catálogo exhaustivo de habitantes de América, sino en la jerarquización o prelación de estadios evolutivos que expliquen circunstancias actuales. Para ello dicen, hay que empezar a contextualizar las muestras.

En la miscellanea incluida en el American Anthropologist de julio-septiembre de 1913 (reporte idéntico al impreso por Science ese mismo otoño), se incluye una nota sobre la Escuela Internacional de Arqueología y Etnología Americana en la ciudad de México, cuya intención es hallar concierto entre las directrices para la investigación que ensayaron Eduard Seler (1909-10|Berlín), Franz Boas (1910-11|Columbia), George C. M. Engerrand (1911-12|México) y el más reciente Alfred Marston Tozzer (1912-13|Harvard). Y el convenio es justamente ese formalismo en la curaduría de los materiales recolectados para su entrega y disposición por el museo mexicano. Evidencia de ello es el Álbum de Colecciones Arqueológicas que compila Manuel Gamio bajo la tutela de Franz Boas. Publicado hasta 1921, las ilustraciones son de Adolfo Best Maugard.

Trabajando para la Secretaría de Educación Pública había sido encargado por el gobierno de coordinar la celebración del Centenario de la Independencia. Su tarea consistía en efectuar un festejo tan distinto como fuera posible del que se había llevado a cabo en 1910. Best Maugard organizó una "Noche Mexicana": una serie de eventos que se llevaron a cabo en Chapultepec, en el lago y a los pies del Castillo. Artes populares, comida típica y diversas manifestaciones folclóricas, a la manera de un "tianguis". El tema "indio" fue explotado a conciencia y se retrató la verdadera "esencia" del México cotidiano. El presidente Álvaro Obregón inauguró la Exposición de Arte Popular Mexicano el 19 de septiembre de 1921, allí se hizo oficial que había un "genio" y habilidad indígenas claras en 
la tradicional producción de artesanías y cuya naturaleza era de hecho artística (22). Al año siguiente Miguel Covarrubias organizaría otra celebración de folklore mexicano, esta vez ayudando al antropólogo Alfonso Caso (23). Sería Gerardo Murillo el Dr. Atl quien hiciera hincapié en el hecho de que concentrándose en "artes precortesianas" el gobierno estaba de hecho dejando de lado y de manera negligente toda una tradición vernácula y que al parecer no se consideraban dignas del templo cívico que es todo museo (24). Ésta versión que agregará artesanías al entramado de la Mexicanidad se construye también por extranjeros que visitaban y estudiaban nuestro país. Sergei Eisenstein filmó hacia 1931 ¡Que viva México!, Anita Brenner había escrito su Idols behind the altars para 1928, mientras que Jean Charlot advertía a los mexicanos de la importancia de José Guadalupe Posada (25).

La ecuación tripartita de RivaPalacio para la historia de México está entonces asumida y es materia de libros de educación primaria con José Vasconcelos y Lázaro Cárdenas. Inclusive la batería de autoridades preferida para explicar al museo es la de Riva Palacio, Jaime Torres Bodet define el 27 de septiembre de 1944 para Ávila Camacho al Museo Nacional de Historia de la República Mexicana: "conforme al pensamiento de Diderot... dedicado a enseñarle al público a ver... es órgano de la cultura nacional..." ${ }^{\prime 26}$. Cuando el 18 de septiembre de 1947, al inaugurarse el Museo Nacional de Artes Plásticas por el presidente Miguel Alemán, la exposición mostrada responde al canon decimonónico que explicaba la historia mexicana $\left(\mathrm{N}^{\prime}+\mathrm{R}^{\prime}+\mathrm{C}^{\prime}=\mathrm{M}^{\prime}\right)$ : “... arte precortesiano...el famoso Ehécatl, Xochipilli, Xilonen ...45 autorretratos de pintores mexicanos de los siglos XVIII y XIX y otras obras representativas de los mismos artistas...jóvenes contemporáneos: Guillermo Meza, Juan Soriano... pasando naturalmente por Rivera, Orozco y Siqueiros ..."27. Como Jefe del Departamento de Bellas Artes en el Instituto Nacional de Bellas Artes, Fernando Gamboa le explicaba a unos reporteros, porqué había estructurado aquella exposición en tres secciones: "auto-retratos de los siglos XVIII y XIX; jóvenes artistas contemporáneos y artes pre-Cortesianas"(28). Se trata de la misma narrativa y sucesión de grupos temáticos perfeccionada y llevada al extranjero después de la Bienal de Venecia en 1950. Margarita Nelken escribió una serie de artículos reseñando las diferentes secciones de las que se componía la exposición. En ellos subrayaba esas "razones para sentir orgullo... y (en) un sentido de perspectiva histórica, la continuidad ininterrumpida de la evolución en nuestro arte... así en lo pre-hispánico como en el futuro inmediato" (29). La Exposición de Arte Mexicano, Antiguo y Moderno (EAMAyM) era el modo más apropiado para mostrar esa peculiaridad de la cultura en México; se trata de ideas lugar común y de credo compartido para la década del 1940. En el periódico Novedades se hablaba de "grandes manifestaciones artísticas... desde las culturas indígenas hasta nuestros días... una fuerza creativa íntimamente 
relacionada con el pueblo... las secciones de Arte Moderno y Contemporáneo, así como la de Artes Populares, se complementan unas a otras" (30). Gamboa mismo lo explicaba en una conferencia que dictara para el pabellón mexicano en la Biennale de Venecia:"Luego de tres siglos de dominación colonial española, y a mediados del siglo diecinueve, México recuperaba su expresión plástica propia"(31). Es claro que de no ser por modos distintos de llamarlas, estas exposiciones constan de instantes narrativos idénticos (Nacimiento + Resistencia + Consolidación).

Pero ocurrirá entonces un cambio de paradigma y que obedece a una racionalidad vanguardista. Gamboa estaba seguramente al tanto del influyente trabajo de Alfred H. Barr jr. en la muestra inaugural del Museo de Arte Moderno en Nueva York en 1929. Aquella fue la primera vez en que se vio un novedoso y modernista modo para exhibir obras de arte. Se trata de una aproximación a la exposición de objetos, que los aísla para ser blanco de una contemplación estética, los separa de su contexto original y obliga cierta lectura unívoca. La clase de exposición que habían intentado René D'Harnoncourt y Alfred H. Barr jr. constituirá el paradigma "atemporal" (timeless). Dicho patrón está orientado hacia una interpretación del espacio que ubica obras limpiamente enmarcadas, al nivel del ojo y en muros neutros, de modo tal que el conjunto todo se halla estetizado (32). Para relacionar aquellos objetos conceptualmente hacían falta las cédulas y el argumento académico generalizador de la exposición en su conjunto. ${ }^{33}$ Cuando Gamboa montaba la exposición The Art of José Guadalupe Posada en el Art Institute de Chicago, presentada en 1944 (abril 13- mayo 14, 1944), leyó con interés un artículo escrito por Frederick J. Kiesler. Este le fue dado por Daniel Catton Rich, Director del instituto en Chicago quién también le pasó el boletín de la American Association of Museums y un artículo sobre construcción de museos en los EUA. ${ }^{34}$ Para entonces tan reciente como 1942, titulado Notes on Designing the Gallery, explica las conjeturas y convicciones de Kiesler sobre cómo el hombre primitivo no distinguía entre visión y realidad fáctica. ${ }^{35}$ En resumen dice que, cuando éste sujeto ancestral pintaba en las cavernas o tallaba esculturas, no había marco o soporte alguno para dicha ilustración del mundo que lo rodeaba. No había distancia entre la escena y su representación. La realidad y su copia no aparecían para el hombre primitivo como entidades o fenómenos separados y distintos. Notes on Designing the Gallery es un documento de una página jamás publicado y que se preservó en los Archivos del legado Kiesler. ${ }^{36}$ Pero Gamboa tuvo siempre consigo el documento en su oficina. ${ }^{37}$ Kiesler defendía su idea de la "unidad primordial", teorizando que la obra de arte que reconocemos actualmente en aquellas cuevas y tallas tenían una función activa, es decir, se trataba de factores orgánicos de la vida humana, no de una liminalidad estética o de mercancías. En menos palabras, el arte era a la 
vez sagrado y social, evento para la veneración y eje de la vida en comunidad. Carecía de un lugar más allá de estas funciones concretas en la cotidianeidad de las tribus primigenias. Esa elaboración es similar al concepto de "arte" que estetas e historiadores contemporáneos de Gamboa componían sobre el pasado mexicano. Entre otros, Justino Fernández a propósito de la Coatlicue y Miguel Covarrubias con sus esquemas iconográficos para ubicar a la cultura Olmeca. ${ }^{38}$

Un importante llamado de atención para otro cambio de paradigma -ahora "contextualizante"- lo constituye la visita de Paul Rivet (Director del Musée de I'Homme en París) a México en agosto de 1938. Jorge A. Vivo reseña en El Nacional y dice: "nos sirvió... [para] no hacer de cada rama de la antropología una entidad independiente y desvinculada del resto...[debíamos] recurrir siempre a fuentes lingüísticas, antropofísicas y culturales..." ${ }^{\prime 39}$. Alfonso Soto Soria recordaba que en 1943 cuando estudiaban Museografía con Rubín de la Borbolla, Rivet y su ideario eran de lo más discutido. Aquellas esculturas prehispánicas y las artesanías actuales debían ahora contar con un tinglado escenográfico que imitara su entorno primero. Sin embargo superar el referente indígena como quinta esencia de lo mexicano era más complejo que la adopción de un modo nuevo de organizar exposiciones al público. Cuando en el marco de las discusiones para armar una exposición rumbo a Bruselas 1958, Pedro Ramírez Vázquez entrevistado por Luis Suárez sugiere que siendo sólo el $10 \%$ de la población actual ya no conviene incluir sus expresiones artísticas o hacer énfasis en su realidad, Alfonso Caso lo regaña públicamente desde el Novedades ${ }^{40}$.

Este concepto extrapolado de México como realidad representada en el museo y fundamentalmente indígena, es también la cancelación ontológica de toda realidad objetiva para el país, dado que tal significado ha sido secuestrado por la alegorización poética que la historia de la nación explicada e ilustrada por el museo implica. 
(ENDNOTES)

1 Donato, Eugenio "The Museum's Furnace: Notes toward a contextual reading of Bouvard and Pècuchet" en Harari, Josué V. ed. Textual Strategies. Perspectives in Post-Structuralist Criticism Cornell University Press, Ithaca, 1979, p. 220-221.

2 Nettie Benson papers collection U'Texas, Austin --- Report on the meeting of the Junta for the Ramo de la Seda; elections: Ministro de la Suprema Corte de Justicia Don José María Bocanegra to be president; Mariano Riva Palacio, Vice-President; General Ignacio Basadre, Secretary; Ignacio Cumplido, Secretary; Lorenzo Carrera, Treasurer; Ignacio Cortina Chávez, Contador; Juan Durán, Benito Quixano, and Colonel Tiburcio Cañas to the Comisión Censora; Isidro Rafael Gondra, General Manuel Céspedes, Esteban Guénot, Lic. Francisco Olaguíbel, and Colonel Gerónimo Villamil to the Comisión para redactar el reglamento. Next meeting to be held at the University. -- Mexico, March, 1841. --*Announcement of meeting of the Empresa General para Explotación del Ramo de la Seda at the Museo Nacional. 1144 Mexico, ALS. April 23, 1841. 1 I. $15 \times 21$ cms. 1145 Vázquez, José María. Chalco, ALS. April 25, 1841.

3 LaChinaca, 16 abril 1862 -op. Cit. Díaz y de Ovando, C. pp. XX

4 Richards, Thomas "Archive and Utopia" en Representations no. 37 Special Issue: Imperial fantasies and PostColonial Histories (Invierno, 1992) pp. 104135.

5 Thomas Richards define al "complejo archivístico" en términos de un espacio utópico para el conocimiento comprensivo y que ubica un dominio geopolítico, el centro de todo conocimiento posible sobre la nación, la librería última y biblioteca de bibliotecas, el museo final. Véase; "Archive and Utopia" en Representations No. 37, Special Issue: Imperial Fantasies and Postcolonial Histories (Winter, 1992), p. 104-135.

6 Hale, Charles A. The Transformations of Liberalism in late nineteenth-century Mexico Princeton University Press, New Jersey, 1989, pp. 3-19 
7 "Libertad, Orden y Progreso" era el lema de los mexicanos tras 1876 y como precondición a la reconstrucción cívica de la nación. Véase; Rodríguez, $\mathrm{O}$. ed. The divine charter: Constitutionalism and Liberalism in nineteenth-century Mexico, Rowman \& Littlefield Inc., USA, 2004, 464 p. Reyes Heroles, Jesús La historia y la acción; la revolución y el desarrollo político de México, México, ed. Oasis, 1978, xxiv-301.

8 Riva-Palacio, Vicente [comp.] et al México a través de los siglos: Historia General y completa del desenvolvimiento social, político, religioso, militar, artístico, científico y literario de México desde la antigüedad más remota hasta la época actual, Espasa y compañía, Barcelona, 1887-1889; 5 vols.

9 Dependiendo el contexto histórico (Reforma, Porfiriato o tiempos post-Revolucionarios), la resistencia (contra los EUA en 1847, contra Francia en 1862, Americanos otra vez en Veracruz en 1914) y la consolidación cambiarán también, pero el nacimiento sigue siendo la Conquista toda vez que esta narrativa se considere.

10 Luis González y González está de acuerdo en que el "nacionalismo aparece como la raíz de nuestra historiografía... las invasiones extranjeras refuerzan este sentido de conciencia propia..." en R. Reyes, ed. Obras Completas de Luis González y González, México, Clío eds. El Colegio Nacional, 2000, (vol. XVI De Maestros y Colegas) pp. 13-19.

11 Lucas Alamán, "Iniciativa para la administración del Museo y Jardín Botánico", en "Memoria de la Secretaría de Estado y del Despacho de Relaciones Interiores y Exteriores leída por el Secretario del Ramo en la Cámara de Diputados el día 12 de febrero de 1830 y en la de senadores el día 13 del mismo", en Lucas Alamán, Documentos diversos, México, Jus, 1945, T. I, p. 240

12 Tenorio Trillo advierte que para 1900 era común entre los mexicanos ubicar como nodales en la historia nacional a tres héroes: "Hidalgo por la Independencia, Juárez por la Libertad y Díaz por la Paz". Quien había iniciado ese dispositivo tripartito que explica el devenir de la nación fue Riva Palacio con la "beatificación del último emperador indio, Cuauhtémoc, la figura del presidente Juárez" y un énfasis en ese vacío que Díaz llenaba, los puntos suspensivos que la circunstancia política actual guardaba. 
13 Buxó, José Pascual "El sueño de la patria nueva. Riva Palacio y la Exposición Internacional Mexicana de 1880" en Revista de la Universidad México, no.4 2004 pp. $91-96$

14 Sistema Nacional de Fototecas INAH México fotografía número de serie 358788.

15 En la Benson LatinAmerican Collection de la U' de Texas en Austin además, están los papeles de V. RivaPalacio tres de cuyos legajos (G582-3, G604) llevan los sugerentes nombres: "Evolución rutaria y apéndices cutáneos de la raza indígena pura"The native races of America" e "Historia General de México. Prospecto".

16 Rutsch Zehmer, Mechthild I.M. Antropología Mexicana y Antropólogos Alemanes en México desde finales del siglo XIX hasta principios del siglo XX Doctorado en Antropología, tesis; UNAM-F.F.y L, Instituto de Investigaciones Antropológicas, 2002, pp. 1-25.

17 Mendoza y Sánchez, G. [1882] Guía del Catálogo de las Colecciones del Museo pp. 446, como se cita en Rutsch op.cit. pp. 37-39

18 Rico Mansard, L. op. cit. ibid - document reproduced in Appendix I, p. 12

19 Ley sobre Monumentos Arqueológicos [1897] en la Constitución de los Estados Unidos Mexicanos México, Imprenta del Gobierno Federal, 1911, 454 pp.

20 Seler, G. Eduard "Bases y fines de la investigación arqueológica en el territorio de la República Mexicana y países colindantes" en Reporte 1910-1911 Escuela Internacional de Arqueología y Etnología Americanas Publicaciones de la EIAEA Imprenta del Museo Nacional; México, 1912; pp. 26

21 Archivo General de la Nación; ramo Instrucción Pública y Bellas Artes AGN/ IPBA caja 168 exp.44 folios 20-23

22 El Universal 20 Septiembre de1921 vol.19 no.1832 año 5 pp. 1-ss. 
23 Williams, Adriana Covarrubias D. Ober, ed. University of Texas Press, Austin, 1994; pp.13-16.

24 Murillo, Gerardo Atl se quejaba del Departamento Etnológico del Museo Nacional Las artes populares... ibid op. cit. pp.45

25 Se trata de cuarenta y un grabados en madera, obra de Hans Holbein "El Joven", tomadas de un facsímil de la edición francesa de 1538.

26 Excelsior 28 IX 1944 año XXVIII tomo V no. 9923 pp. 8

27 El Nacional 18IX1947 no. 6643 2a época año XVIII tomo XXIV pp. 4

28 "Inaugurado el Museo Nacional de Artes Plásticas" en El Nacional Jueves 18 Septiembre, 1947 México $1^{\mathrm{a}}$. sec. pp.1 y 4; año 28 volumen 24 no. 6643; 2a. época

29 Nelken, Margarita "La Gran Exposición de Arte Mexicano" en Excelsior domingo 6 diciembre, 1953 no. 13225 pp. 9 secc. B año 37 vol. 6 (también en los números 13210 y 13217 , Noviembre 23 y 30)

30 Gamboa, Fernando "La Exposición de Arte Mexicano en París" en Novedades Agosto 171952 pp. 8-ss: "... las grandes manifestaciones artísticas mexicanas...desde las culturas indígenas y hasta nuestros días... fuerza creadora en estrecha relación con el pueblo... las secciones de Arte Moderno y Contemporáneo y Arte Popular se complementan."

31 Gamboa, Fernando n/d Mayo 81950 documento mecanografiado folio 48TT ocho páginas Archivo Fernando Gamboa.

32 Marquis, Alice Goldfarb. Alfred H. Barr, Jr.: Missionary for the Modern Chicago, Contemporary Books, 1989; passim -219 pp.

33 Staniszewski, Mary Anne The Power of Display. A History of Exhibition installa- 
tions at the Museum of Modern Art The MIT Press, Cambridge \& London, 1998, pp. 61-66.

34 Vail, Lawrence "Recent Museum Building Experience in the USA" The Museum Journal vol.47 pp.221-227 Marzo 1948 (documento conservado en el Archivo Fernando Gamboa con el folio FG-97)

35 Kiesler, Frederick "Notes on Designing the Gallery" (1942) Archivo Fernando Gamboa, documento FG-6 dice: "Today the framed painting on the wall has become a decorative cipher without life and meaning, or else, to the more susceptible observer, an object of interest existing in a world distinct from his. Its frame is at once symbol and agent of an artificial duality of "vision" and "reality" or "image" and "environment," a plastic barrier across which man looks from the world he inhabits to the alien world in which the work of art has its being. That barrier must be dissolved: the frame, today reduced to an arbitrary rigidity, must regain its architectural spatial significance" Una transcripción se halla disponible también en www.arch.mcgill.ca/prof/mellin/arch671/winter2001/dkouba/drm/kiesler.htm URL accesado 02/02/2004, 19:19 GMT

36 Kiesler, Frederick "Notes on Designing the Gallery," unpublished manuscript cited in Melvin Lader, Peggy Guggenheim's Art of This Century: The Surrealist Milieu and the American Avant-Garde, 1942-1947, tesis doctoral para el departamento de Historia del Arte en la Universidad de Delaware, EUA, 1981 , pp. 114.

37 Archivo Fernando Gamboa. doc. FG-6 una página mecanografiada, copia al carbón.

38 Fernández, Justino Estética del arte mexicano: Coatlicue, el retablo de los reyes, el hombre, México, UNAM, 1972, 599 pp.; y Covarrubias, Miguel Origen y desarrollo del estilo artístico Olmeca México, 1942 Sociedad Mexicana de Antropología; (Reuniones de la Mesa Redonda: Mayas y Olmecas. 46-49)

39 El Nacional 17VIII'38 no. 3347 2a época año X tomo XVI pp. 3

40 Novedades (27 abril 1958 no. 6209 año XXIII "México en la Cultura" no. $4762^{\text {a }}$ época - p.p.) 
